All mammals are provided with two distinct adipose cells: white and brown adipocytes. White adipocytes store lipids to provide, in time of need, energy to the organism and release hormones and cytokines involved in energy homeostasis whereas brown adipocytes controls energy expenditure using lipids to produce heat. Previous descriptions implied their localization in distinct sites of the body, but our and other studies demonstrated that they are present together in many fat depots in murine adipose organ. This observation, combined with anatomical data and signs of physiological interaction of the two cell types in specific experimental conditions, gave rise to a new concept: the adipose organ. The cohabitation of white and brown adipocytes could be explained with the hypothesis of reversible physiological transdifferentiation within the adipose organ; they are able to convert one into each other. If needed, the brown component of the organ could increase at the expense of the white component and vice versa. This plasticity is important because the brown phenotype of the organ associates with resistance to obesity and its related disorders. Recently, the presence of metabolically active, uncoupling protein1 immunoreactive, brown adipose tissue (BAT) has been disclosed also in adult humans. The distribution of brown adipocytes into the human adipose organ seems to replicate that of murine adipose organ. The amount of BAT inversely correlates with the BMI of patients suggesting a role in the energy balance of humans. Moreover, cells with features of brown adipocyte precursors were found in pericapillary areas. Future therapeutic strategies for the treatment of obesity and related disorders could include maintenance of brown adipocytes, stimulation of the growth of pre-existing brown precursors or induction of trans-differentiation of white into brown adipocytes.

\title{
Climate change and obesity
}

\author{
João Breda
}

Senior Officer Noncommunicable Diseases and Environment (Nutrition), WHO Regional Office for Europe

The actual relations between energy, food and health are enormous, raising the need to discuss very important policy options. The double burden of disease associated with malnutrition (undernutrition to obesity) continues to grow. Poor diet, overweight and obesity contribute to a large proportion of non-communicable diseases, including CVD and cancer. Food habits have been changing dramatically in many countries and tendencies have been identified like the excessive salt, sugar and fat intake, low fruit and vegetable intake and an increasing problem of obesity. Recent life cycle assessment and carbon footprint studies of food products have clearly shown that differences in environmental impacts between different food products are tremendous, and that various products affect in different environmental problems. According to environmental accounting in the food sector, the food chain accounts for $7 \%$ of $\mathrm{CO}_{2}$ emissions, $43 \%$ of $\mathrm{CH}_{4}$ emissions and $50 \%$ of $\mathrm{N}_{2} \mathrm{O}$ emissions produced across the entire economy. It is widely accepted that agriculture, especially livestock production, accounts for about a fifth of total greenhouse gas emissions, therefore contributing to climate change and its adverse health consequences. From the analysis of greenhouse gas emissions and energy consumption during the life cycle of carrots, tomatoes, potatoes, pork, rice, etc. one can find huge differences in terms of the contribution of each food.
Also some researchers have been developing comparisons of different meals composed of different food items and its impact. An interesting example is that, for instance a meal with tomatoes, rice and pork has nine times higher emissions than a meal made from potatoes, carrots and dry peas. Another issue is the physical activity. Some argue that if the European population should return to the habit of walking and cycling to work replacing cars. Some argue that this would have a significant impact on obesity and a substantial impact on emissions. Effects of climate change on health and nutrition will affect mostly children and the lower socio-economic layers of the society. There is strong evidence that climate will deepen inequalities. There is also a discussion on the contribution of those who are overweight contributing more to the climate change because allegedly they consume more energy, bigger portion sizes and overall increase food consumption especially from those categories that contribute more to climate change. This perspective is based on individual approach and responsibility only and places the burden and more stigmatization on the overweight and obese individuals. There is also a very interesting argument in favour of breast-feeding in relation to climate change. By enabling more women than currently doing so to exclusively breast-feed their children for the first 6 months of life, some argue that there could be a reduction of the 
number of children requiring attention for overweight due to the protective effect of breast-feeding. There would be less need for the diversion of foodstuffs through dairy animals to produce breast milk replacements, and less need for the use of materials and energy. Producers of animal products, food consumers decision makers in public catering are the critical stakeholders for minimizing climate change impacts of the food chain. From a household's point of view food chain constitutes one of the largest source of greenhouse gas emissions in the EU countries, together with mobility and residential energy use. The health sector is critical to the development of comprehensive approach to the mitigation of climate change-related aspects. The adaptation of the traditional health and public health approaches to make them sensible to climate change is an issue worth attention. The responses in the area of food and nutrition in relation to climate change need to be developed, implemented and fine-tuned. The WHO policy framework based on the commitments of the European Charter to Counteract Obesity and the European Food and Nutrition Policy Action Plan 2007-2012 both highlight the importance of measures involving the food chain to prevent overweight and obesity. Some authors consider important these priorities in responses to the challenge posed by climate change and nutrition with a focus on children. WHO is developing specific projects on climate change and nutrition in some countries of the region.

\title{
Health and environmental benefits from combined control of obesity and climate changes
}

\author{
R Viscecchia ${ }^{1}$, A Stasi $^{1}$ and M Prosperi ${ }^{2}$ \\ 'Dept. PrIME, University of Foggia, Foggia, Italy: ${ }^{2}$ Department of Bioagromed, University of Foggia, Foggia, Italy
}

Introduction: Modern lifestyle is one among the most relevant causes of obesity and climate change. Preventing both of them implies a win-win strategy for the improvement of social wellness (i.e. health and environmental benefits). A suitable strategy could be addressed to the partial substitution of caloric food (e.g. meat), which is also responsible for a large amount of greenhouse gas (GHG) emissions, with less caloric food (e.g. fruits and vegetables), which is proved of exerting a lower impact on climate change.

Method: The analysis is referred to the Italian case study, based on data from the Italian Statistical Institute. The relationship between obesity and climate change is here explained experimentally through a two-step model. In the first step, a regression model (Ordinary Least Squares method) is adopted to explain obesity rate in terms of ratio of people consuming meat more than once a week, and the ratio of people consuming fruits and vegetables less than once a day. The outcome of this analysis allows defining a policy target in terms of obesity abatement, provided by a reasonable change in food demand. In the second step, the observed change in food demand from the previous step is combined with Life Cycle Analysis indicators, retrieved from the scientific literature, referred to each type of food (i.e. meat, fruits and vegetables) in order to evaluate the impact of food industry on climate change.
Results: The Italian population shows alerting diet habits, with about $70 \%$ of people consuming meat at least once a week (first group), implying an excess of energy intake. In addition, about $56 \%$ of people are not used to eat fruits and vegetables at least once a day (second group). Assuming a policy aimed at cutting by half the ratio of both groups, by using the first model we estimated a reduction of the obesity rate from the current $9 \cdot 68 \%$ to $7 \cdot 04 \%$. This implies an increase in the annual consumption of fruits and vegetables by $1668000 \mathrm{t}$, and a reduction of $873360 \mathrm{t}$ of meat. By using the second model, we estimated a significant reduction of $\mathrm{CO}_{2}$ emissions (used as a proxy of GHG), by $5406000 \mathrm{t}$ per year. In economic terms, the effect of the policy can be roughly estimated as 26.7 MEur of benefits for reducing the expenditure in obesity prevention, and 85.58 MEur of gain due to the reduction in carbon emissions.

Conclusions: The study shows the evidence that the consumption of food with low content of energy has a twofold effect in the reduction of obesity and in the mitigation of climate change. The approach developed in the present study highlights the opportunity to design several measures within climate change policies targeted at the promotion of healthier and more environmentally oriented diets. However, there is still room for discussion on the design of effective and efficient strategies for a new dietary behaviour. 